\title{
Mapping the Intellectual Progress in e-Business, e-Services and e-Society from 2001 to 2019
}

\author{
Zacharoula Papamitsiou ${ }^{(\bowtie)}($ (D) and Patrick Mikalef (D) \\ Norwegian University of Science and Technology, Trondheim, Norway \\ \{zacharoula.papamitsiou,patrick.mikalef\}@ntnu.no
}

\begin{abstract}
This study aims to identify the conceptual structure and the thematic progress in e-Business, e-Services and e-Society and to elaborate on backbone/emerging topics in the field from 2001 to 2019. To address this objective, this paper employs hierarchical clustering, strategic diagrams and network analysis to construct the intellectual map of the I3E community and to visualize the thematic landscape in this field, using co-word analysis. Overall, a total of 835 papers from the proceedings of the e-Business, e-Services and e-Society (I3E) conference, and the respective 2574 author-assigned keywords, were included in the analyses. The results indicate that the community has significantly focused in areas like technology adoption models, Social Media, e-Government and business models; sentiment analysis and m-payments are peripheral themes, yet topics like cloud computing and Open Data are emerging. The analysis highlights the shift of the research interest throughout the past decades, and the rise of new topics, comprising evidence that the field is expanding and evolving. Limitations of the approach and future work plans conclude the paper.
\end{abstract}

Keywords: Co-word analysis $\cdot$ Bibliometrics $\cdot$ Conceptual mapping $\cdot$ e-Services

\section{Introduction}

The subject area of e-Business, e-Services and e-Society has delved into the topic of digitalization with regards to different facets of how we live, work, and conduct business [8]. Since the early 2000s when the dot com period hit its peak, the research interest has centered on the opportunities, challenges and implications that novel digital technologies introduce. The proliferation of such technologies, coupled with the rapid adoption of computer-based systems and the investments in network infrastructures, created a new wave of opportunities on digitalized processes [1, 22]. Along this progression, research has focused on issues related to each transition, examining obstacles of adoption from the individual to the industry level, value generating mechanisms, and the anticipated and unanticipated effects of integrating these technologies in everyday life.

Now that the field counts 18 years, based on the number of the e-Business, e-Services and e-Society (I3E) conferences being organized, it is a good time to analyze 
its past and current state. Doing so will allow researchers to understand the evolution of the field over the past two decades and identify the challenges and opportunities that lie ahead. The most appropriate way to examine all publications presented in the conferences throughout these years is by applying a methodology that quantifies the core topics, the marginal contributions, the under-developed themes, and the forthcoming ideas that worth investing on, as well as how these topics are related and move between these states during the last 18 years. The main objective of this work is to capture, interpret and understand the big picture in the I3E domain from a quantified viewpoint. The research presented in this paper is a mapping study of the I3E field; it is a review that seeks to identify, not results, but linkages, shows the internal dynamics and structure of the domain, and pinpoints the topics with impact in the given discipline.

Towards facilitating this objective, this paper employs co-word analysis, and examines the associations and networks among concepts, ideas, and issues that have contributed to the evolution of the field to date [5]. Co-word analysis allows for and supports the identification of key patterns and trends that point to particular changes in research topics (e.g., emerging or declining interests) or specific research directions (e.g., paradigm shifts), using a graph of key-terms [11], extracted directly from the metadata of the papers. Considering this, the present study maps the intellectual progress of the e-Business, e-Services and e-Society landscape, as reflected in the records of $\mathrm{I} 3 \mathrm{E}$ conferences. The proceedings of this event provide a solid foundation to the related work published to date. During the past 18 years, considerable work has been published, allowing us to observe where the field currently stands, what are the challenges and opportunities the researchers are facing, and what are the potential driving forces in the near future. Accordingly, this work mainly contributes as follows:

- brings new insights on the intellectual mapping and progress of the area of I3E;

- raises awareness of the community on the mature, under-developed, emerging, or declining research themes;

- highlights individual topics as popular, core or backbone topics within the discipline.

\section{Background and Related Work}

The community of I3E is inherently diverse at the intersection of digital technologies, business, social sciences, big data, artificial intelligence, and network infrastructures $[1,8]$. Digital services can make a global impact and therefore, the understanding of the economic, technical, and social aspects of service development and innovation is a demand [16]. The quantity and quality of the research activity within this community has been the topic to a variety of literature reviews, aiming to evaluate the research progress, impact and societal value, from different viewpoints (e.g., [9, 12, 26, 27]).

For instance, persuasiveness of electronic Word-of-Mouth (eWOM) communications has received much attention from scholars because it can affect consumers' purchase attitudes, intention, decisions, and hence sales. A dedicated review of existing literature on the topic offered an overview of the determinants of eWOM persuasiveness, identified gaps in current research and provided directions for future research [12]. 
Another study reviewed inclusive eGovernment strategies and socially-aware eGovernment policies, and highlighted the need for a better understanding of the role of new intermediaries as actors that can impact the qualities of the citizens' and publicsector relationships [26]. The results recognized the usefulness of placing the intermediaries in an institutional framework and the authors proposed an agenda for future research.

More recently, the emergence and extended discussions about blockchain and what it can offer to businesses in relation to consumers (B2C), businesses (B2B) and governments (B2G) were under the lens of a systematic literature review of 40 articles from business, management and accounting peer review journals [9]. The findings demonstrate the role of blockchain as a facilitator of instant payments, trusted interfaces and traceability of goods for the consumers. Specifically, businesses can be benefited from blockchain in terms of machine-to-machine transactions, accounting, business process management and provenance traceability. New business opportunities also arise in government sector such as digital storage, authentication and maintenance of records, smart trust codification and new market for digital payment services and global commerce [9].

In another systematic review of 71 articles, social media applications over the marketing context were explored [2]. The study synopsized the main themes and trends, including the role of social media on advertising, the eWOM, customers' relationship management, and firms' brands and performance.

Apparently, despite the several literature reviews in the field, they are sparse and there is no previous attempt - to the best of our knowledge - that maps the field as a whole. As I3E is a highly diverse and continuously evolving field, it is important to (a) identify and understand its core foundations that might contribute to reinforcing the community's identity; (b) detect under-represented or under-developed themes that require attention for their inclusion and success; (c) highlight research gaps in bridging theory and practice; and (d) find challenges and opportunities that hold the promise for improving the digitization processes. As the digitalization process and its outcomes in the 21 st century accelerate transformation and the creation of sustainable societies, offering tremendous opportunities for revising current business methods and practices [22], there is a critical need for understanding and evaluating the field as a whole.

\section{Methodology}

\subsection{Data Collection}

The data analyzed in this study were downloaded from the I3E Springer proceedings between 2001 and 2019. Overall, 835 peer-reviewed papers were produced within the community of I3E and published to-date. The keynote speeches, prefaces to the conference-, or track proceedings, as well as the papers that did not contain any keywords, were excluded from the analysis. From the collected papers, the author-assigned keywords were extracted from the metadata of each paper and were used as a unit of 
analysis. The keywords used for the description of the content of a publication can be seen as the basic building blocks of the structure of a research field; an article's keywords provide an adequate summary of its content, and thus can be utilized to reduce a large space of descriptors (i.e., article text) to a network graph of smaller related spaces (i.e., keywords) [6]. Although when authors choose keywords to describe their work those keywords can either be very generic or very specific, still, those keywords are human annotations on the content of the papers, reflecting human judgement and perspective. The idea is to understand the conceptual structure and evolution of a field directly from the interaction between keywords: if two keywords co-occur within a paper, then the two topics they represent are related; higher co-word frequency implies stronger correlation in keywords pairs, further suggesting that two keywords are related to a specific theme [6]. The 835 papers are distributed per year of publication as shown in Fig. 1.

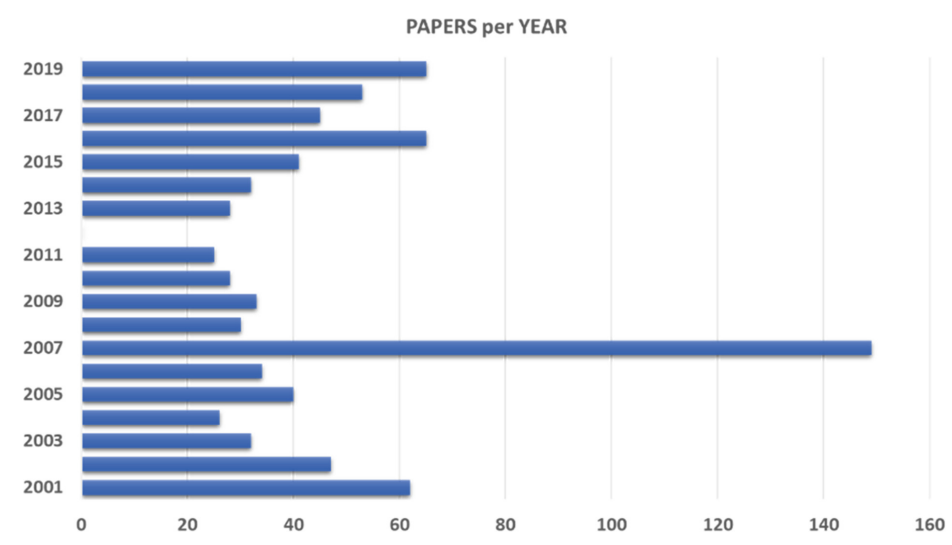

Fig. 1. Number of I3E publications per year for the period 2001-2019.

\subsection{Data Pre-processing}

From the 835 published papers, the 531 had author-assigned keywords. Specifically, 2574 keywords $(M=4.85$ per article) were identified in total. The retrieved authorassigned keywords were manually pre-processed and standardized through merging words that convey similar meaning (e.g., "electronic government" and "e-Gov" were merged into "eGovernment"), fixing misspelled keywords (e.g., "goverment"), following a common spelling for UK and US terms (e.g., "behaviour" and "behavior"), and filtering broadly used terms (e.g., "IoT" and "Internet of Things"; "WoM" and "Word of Mouth") - following the approach recommended in [11, 17], in a noninvasive manner. Keywords appearing in singular and plural forms of nouns and gerunds were also merged. At the end of this pre-processing, 1732 keywords $(67.3 \%$ of the original dataset) were identified as unique and were subjected to further analysis. In order to be able to apply this method, papers from more than 5 years of research are required. 
The Kolmogorov Smirnoff test shown that the frequency of keywords follows a power-law distribution with an alpha of 1.87. Due to this heavy-tailedness, the research landscape of $\mathrm{I} 3 \mathrm{E}$ is a scale-free network, with small number of popular terms acting as "hubs": they connect different topics, capture major research directions and influences in the field, and shape its intellectual structure [11]. A scale-free network also suggests that major research themes can be detected with small subset of popular terms. A previous analysis in the HCI research field demonstrated that less than 100 keywords are enough to describe the intellectual progress of a field [17]. Thus, in the present study we decided to include only those keywords that appear more than four times ( $n \geq 4$ ) in the period 2001-2019. This decision was grounded on two facets: (a) the frequency of a term reflects its significance for a research community, i.e., the higher the frequency is, the more often the term attracts the researchers' attention/interest; and (b) the retained 65 keywords (total frequency $=646,37.3 \%$ of the total unique keywords) cover $461(86.8 \%)$ of the 531 articles (with keywords) published. Furthermore, for the given datasets of terms and papers, $n=4$ is the minimum term frequency that achieves the highest inclusion of papers in the datasets. For example, for authorassigned keywords with $\mathrm{n} \geq 3$, the retained keywords are $\mathrm{N}=150$ and cover $88.9 \%$ of the papers, whereas for keywords with $n \geq 5, N=57$ keywords, covering $72.3 \%$ of the papers. Thus, with fewer yet highly frequent terms we could satisfactorily describe the I3E network of terms.

\subsection{Co-word Analysis and Strategic Diagram}

This study employs co-word analysis to shed light on the intellectual progress in the I3E field. Co-word analysis has been proposed as a content-analysis technique to map the strength of relations between terms in texts and to trace patterns and trends in term associated-ness [5]. The idea behind co-word analysis rests on the assumption that keyterms identified within an article (e.g., keywords) can adequately describe and communicate the content of that article; the co-occurrence of at-least two keywords in the same article indicates a linkage between the topics, i.e., a "theme" [4]. The main units of analysis are keywords, clusters (i.e., sets of keywords) and keyword networks [17].

Co-word analysis is applied to reduce the broad network of keywords into a smaller network of related topics using graph theory [7]. Graphs consist of nodes that represent the keywords, and links that represent the interactions between the nodes. Given a network of keywords, a combination of clustering, network analysis and strategic diagrams is used to model the conceptual structure of a field and to characterize it [4]. The graph theory concepts employed are centrality (i.e., the strength of the links from one research theme or cluster to others, indicating its significance in the development of the community [17]) and density (i.e., the coherence of a cluster and a measure of a theme's development [10]). Combining centrality and density allows for the creation of two-dimensional strategic diagrams [4] (Fig. 2): the position of a cluster in the diagram corresponds to the importance of the cluster in the whole network (i.e., centrality $-x$-axis) in relation to how well the theme of this cluster is developed (i.e., density $-y$ axis).

As one can observe, Quadrant I (Q1) holds the motor themes (i.e., mainstream themes) that have strong centrality and high density. Quadrant II (Q2) contains themes 


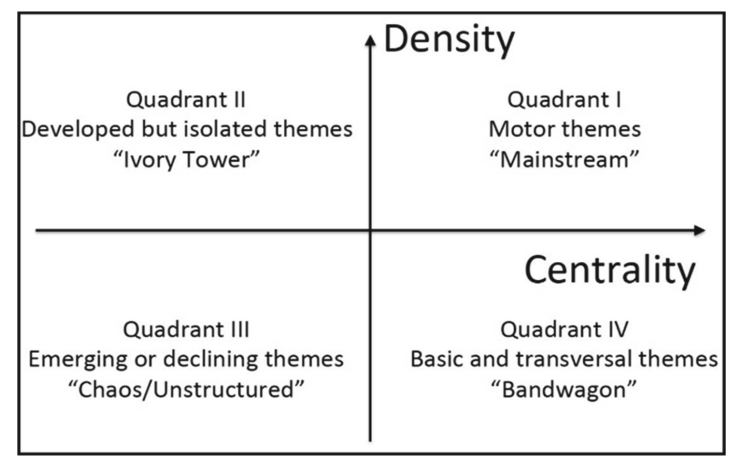

Fig. 2. Number of I3E publications per year for the period 2001-2019.

that are internally well-structured but have weak external ties. These research themes are more specialized and peripheral to the mainstream work that is central in the research field. Quadrant III (Q3) includes the themes with low density and low centrality, that are either emerging, or disappearing. Finally, Quadrant $I V\left(Q^{4}\right)$ covers basic and transversal themes of considerable significance to the entire research network, i.e., central to the community, with potential to become important to the field as a whole.

\subsection{Data Analysis}

To identify the major research themes in the I3E domain, hierarchical clustering analysis on a correlation matrix with the retained terms was performed, using the Ward's method with Squared Euclidean Distance as the distance measurement [19]. The supervised clustering method allows to maintain content validity and cluster fitness for the highest number of clusters $[11,17]$. Each cluster represents a research theme or sub-field. The co-word network was further analyzed using the following measures:

- Keywords: set of terms that constitute a cluster;

- Size: number of keywords in the cluster;

- Frequency: how many times all keywords (in a cluster) appear in the dataset;

- Co-word frequency: how many times at-least two keywords (from a cluster) appear in the same paper. Computing this results in a symmetrical co-occurrence matrix [15]: values in the diagonal cells are term frequencies, and values in nondiagonal cells are co-word frequencies. High frequency of co-occurrence between terms indicates connection between the topics they represent;

- Transitivity: how tightly connected is the cluster (the clustering coefficient), i.e., how close the keywords are to being a "clique". Transitivity is the frequency of loops of length three in the cluster; a loop of length three is a sequence of nodes $x, y, z$ such that $(x, y),(y, z)$ and $(z, x)$ are edges of the graph [25]. The value range is $[0,1]$;

- Centrality: the degree of interaction of a theme with other parts of the network, i.e., how many other clusters a cluster connects to [4]; Centrality refers to a group of metrics that aim to quantify the "importance" of a particular node (or cluster) within a 
network (e.g., betweenness centrality, closeness centrality, eigenvector centrality, degree centrality) [20]. Here we used betweenness centrality (C), with $0 \leq \mathrm{C} \leq 1$;

- Density: how cohesive is the cluster of terms, i.e., the number of direct ties observed for the cluster divided by the maximum number of possible ones [4]. Density is graph-dependent and can be any positive real number [14].

Based on the clustering results, we plotted the strategic diagram for the years 20012019 to visualize the cohesion and maturity of the I3E themes [4, 17]. In addition, a keyword network graph was created from the keywords list. In this graph, each keyword is represented as a node, and the keywords that co-appear on a paper are linked together. By creating associations between keywords, multiple networks associated with different themes are also created. In this case, bridges are built between the nodes of keywords, to allow communication and information flow between isolated regions in the whole network. Those nodes are known as structural holes [21]. Keywords acting as structural holes serve as a "backbone" of a network: if removed, the network will lose its cohesion and will disintegrate into separated and unconnected concepts. Thus, the network's core-periphery structure needs to be computed, to determine which nodes are part of a densely connected core (i.e., with a higher number of bridges), or a sparsely connected periphery [24]. Core nodes are reasonably well-connected to peripheral nodes, while peripheral nodes are sparingly connected to a core node or to each other. A node belongs to a core only if it is well-connected to other core nodes and to peripheral nodes [24]. A follow-up core-periphery analysis was performed to spot the core research topics from the perspective of the whole network. In this analysis, keywords were categorized according to their popularity, coreness (i.e., connectedness with other topics) and constraint (i.e., backbone). The whole approach is illustrated in Fig. 3.

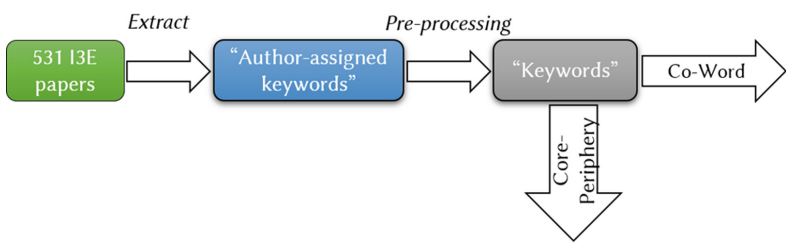

Fig. 3. Research methodology.

\section{Results}

\subsection{Mapping of the Field}

The analysis on the retained 65 author-assigned keywords led to 14 clusters (labeled as $\mathrm{C} 1-\mathrm{C} 14$, in Table 1), with each cluster representing a research theme or a sub-field. In order (a) to better understand the relative "position" of these clusters within the overall I3E field (i.e., what is the distance from each other in terms of cohesion and maturity of 
research themes they correspond to); and (b) to create the conceptual structure of the I3E discipline, we constructed strategic diagrams (plots) using the centrality and density of each cluster [4, 17]. The overall results can be seen in reading Fig. 4 and Table 1 together. In the plots, both axes are centralized to the average centrality and average density respectively (i.e., $0.247,1.361$ ). The overall network's density was 0.667 .

As it can be observed from Fig. 4, one motor theme (Mainstream theme), represented by cluster C5 (i.e., literature review, TAM, adoption) is detected using the human descriptors (keywords) of the papers. In other words, the field is in general fragmented, with only one theme having received substantial attention from the community, in terms of human annotations. Furthermore, in Fig. 4, the author-assigned keywords indicate that the community has few internally well-structured research themes, yet with weak external ties (Ivory Towers), acting as peripheral nodes to the global network (i.e., connect only to core nodes, yet not necessarily to mainstream topics only), and classified in clusters C2, C3 and C4 (e.g., mPayments, Digital Payment, Twitter, Sentiment Analysis, UTAUT2, Perceived Risk). Those topics appear to have high-density, i.e., the clustering coefficient is high and the topics within each of the cluster are very well connected to each other, but they lack strong ties with topics that are external to them. The following-up core-periphery analysis will provide insight on that issue.

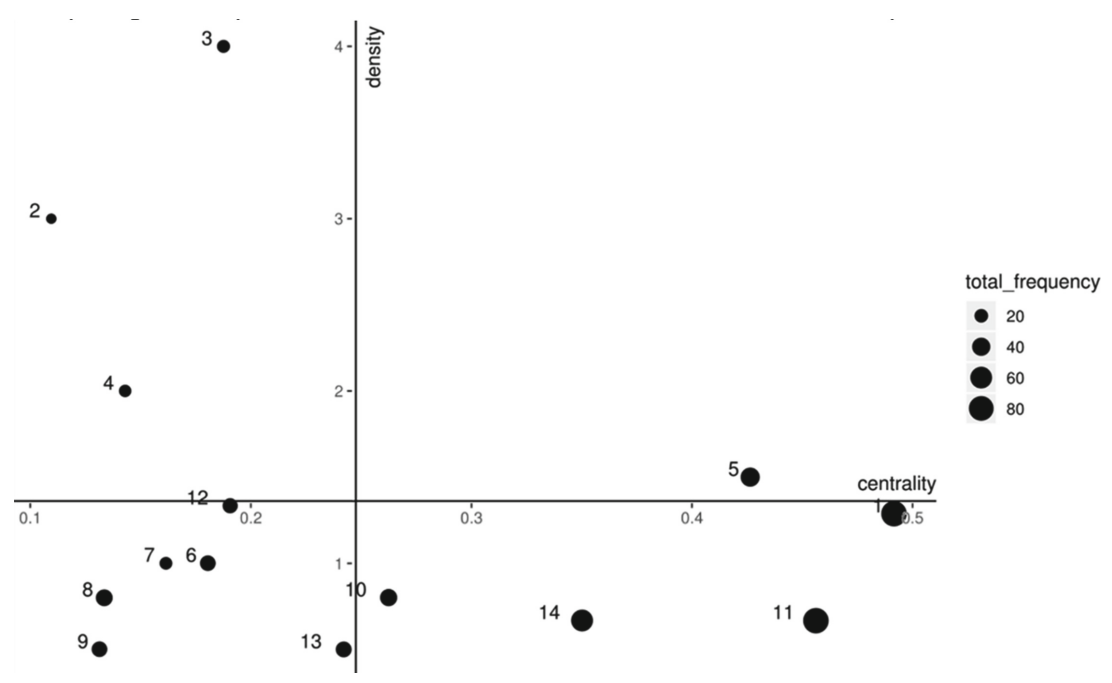

Fig. 4. Strategic diagram for I3E for the period 2001-2019. 
Table 1. Clusters of topics in I3E for the period 2001-2019.

\begin{tabular}{|c|c|c|c|c|c|c|c|c|}
\hline Q & ID & Keywords (the most frequent in bold) & Size & FrEquation $^{\mathrm{a}}$ & $\begin{array}{l}\mathrm{CW}- \\
\text { Fr. }^{\mathrm{a}}\end{array}$ & $\mathrm{T}^{\mathrm{a}}$ & $\mathrm{C}^{\mathrm{a}}$ & $\mathrm{D}^{\mathrm{a}}$ \\
\hline Q1 & $\mathrm{C} 5$ & $\begin{array}{l}\text { adoption, literature review, TAM, } \\
\text { India, use }\end{array}$ & 5 & 45 & 82 & 0.90 & 0.43 & 1.50 \\
\hline Q2 & $\mathrm{C} 2$ & m-payments, digital payment & 2 & 10 & 20 & N/A & 0.11 & 3.00 \\
\hline Q2 & $\mathrm{C} 3$ & twitter, sentiment analysis & 2 & 18 & 27 & N/A & 0.19 & 4.00 \\
\hline Q2 & $\mathrm{C} 4$ & $\begin{array}{l}\text { UTAUT2, information systems, } \\
\text { perceived risk }\end{array}$ & 3 & 16 & 28 & 1.00 & 0.14 & 2.00 \\
\hline $\begin{array}{l}\text { Q2- } \\
\text { Q3 }\end{array}$ & $\mathrm{C} 12$ & $\begin{array}{l}\text { Big Data, data quality, information } \\
\text { sharing }\end{array}$ & 3 & 26 & 27 & 1.00 & 0.19 & 1.33 \\
\hline Q3 & C6 & $\begin{array}{l}\text { eWOM, motivation, e-Services, } \\
\text { information seeking, online reviews }\end{array}$ & 5 & 28 & 39 & 0.87 & 0.18 & 1.00 \\
\hline Q3 & C7 & $\begin{array}{l}\text { Cloud Computing, SaaS, eBusiness } \\
\text { models, business intelligence }\end{array}$ & 4 & 17 & 23 & 1.00 & 0.16 & 1.00 \\
\hline Q3 & C8 & $\begin{array}{l}\text { Open Data, benefits, usability, } \\
\text { evaluation, AHP, public services }\end{array}$ & 6 & 33 & 41 & 0.81 & 0.13 & 0.80 \\
\hline Q3 & C9 & $\begin{array}{l}\text { SME, mobile devices, marketing, } \\
\text { technology adoption, CRM }\end{array}$ & 5 & 28 & 28 & 0.33 & 0.13 & 0.50 \\
\hline Q3 & C13 & $\begin{array}{l}\text { business model, strategy, value, } \\
\text { information technology }\end{array}$ & 4 & 29 & 30 & 0.33 & 0.24 & 0.50 \\
\hline Q4 & $\mathrm{C} 1$ & $\begin{array}{l}\text { Social Media, customer engagement, } \\
\text { Facebook, SNS, ICT, Social } \\
\text { Networks, Use and Gratification } \\
\text { Theory }\end{array}$ & 7 & 86 & 108 & 0.87 & 0.49 & 1.29 \\
\hline Q4 & C10 & $\begin{array}{l}\text { privacy, Social Commerce, WoM, } \\
\text { trust }\end{array}$ & 4 & 35 & 44 & 0.87 & 0.26 & 0.80 \\
\hline Q4 & C11 & $\begin{array}{l}\text { e-Commerce, collaboration, } \\
\text { interoperability, interorganizational } \\
\text { system, security, supply chain, e- } \\
\text { Business, web services, QoS }\end{array}$ & 9 & 86 & 93 & 0.53 & 0.46 & 0.67 \\
\hline Q4 & C14 & $\begin{array}{l}\text { e-Government, Smart Cities, artificial } \\
\text { intelligence, Internet, B2B }\end{array}$ & 6 & 61 & 62 & 0.33 & 0.35 & 0.67 \\
\hline
\end{tabular}

${ }^{\text {a}}$ Freq: Total frequency of all keywords; CW-Fr: Co-word Frequency; T: Transitivity; C: Centrality; D: Density

Regarding the themes that are either emerging or disappearing (Chaos/ Unstructured), the author-assigned keywords revealed that researchers have developed a considerable number of topics with - in a sense - "marginal" interest in the I3E network, classified in clusters C6, C7, C8, C9, C12 and C13 (e.g., eWOM, online Reviews, motivation, information Seeking, eServices, Cloud Computing, SaaS, Business Intelligence, eBusiness models, Open Data, information technology), as illustrated in Fig. 4. The term "marginal" here is used to describe both the cases of "close-to-disappearing" and "nearly rising" topics, i.e., topics that either tend to no-longer attract major interest, or they have recently started to attract attention, but have not yet been well-developed. 
Finally, a substantial number of transversal themes (Bandwagon) have been detected as well, i.e., themes that are strongly linked to specific research interests throughout the network yet are only weakly linked together. These are categorized in the clusters C1 (e.g., customer engagement, facebook, social media, use and gratification theory, social networks), C10 (e.g., social commerce, WoM, trust, privacy) C11 (e.g., eCommerce, interoperability, interorganizational system, security, supply chain, eBusiness, web services, QoS) and C14 (e.g., IoT, Smart Cities, artificial intelligence, eGovernment, b2b).

\subsection{Keywords Network Map}

A network of keywords demonstrates the relationships among different themes; to better understand and visualize the interactions between the research themes in Table 1, network analysis was used to create a granular map of the keywords. Figure 5 displays the results. Each node in the graph represents a keyword that is linked to other keywords that appear on the same paper. The size of the nodes is proportional to the frequency of the keywords, the color of the node corresponds to the cluster the keyword has been classified in, and the thickness of the links between the nodes is proportional to the co-occurrence correlation for that pair of keywords. To reduce visual clutter, a centralized subset of the complete network is illustrated, omitting isolated nodes and keywords with less than 3 strong ties, that would lead to a highly disconnected network.

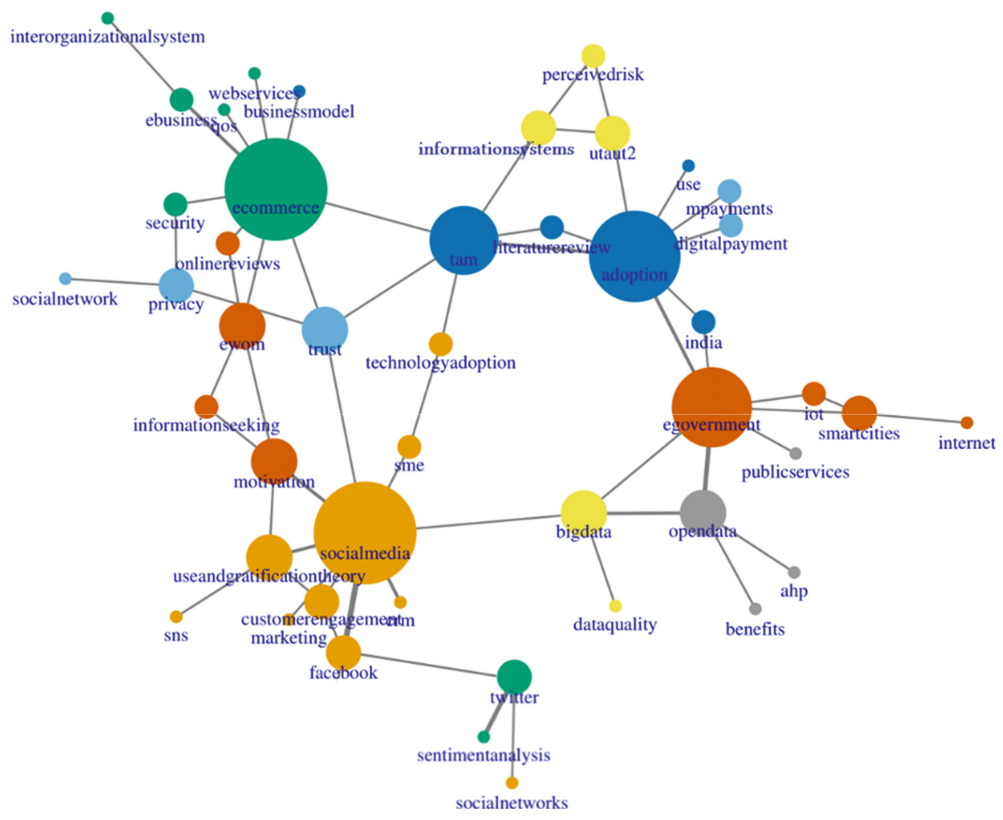

Fig. 5. Keywords network map for I3E for the period 2001-2019. 
Table 2. Summary of popular, core and backbone topics of I3E in 2001-2019.

\begin{tabular}{r|l|l|l|l|l|l}
\hline$\#$ & Popular topic & Frequency & Core Topic & Coreness & Backbone topic & Constraint \\
\hline 1 & Social Media & 51 & Social Media & 0.45 & Social Media & 0.19 \\
\hline 2 & e-Government & 37 & e-Commerce & 0.30 & TAM & 0.19 \\
\hline 3 & e-Commerce & 36 & TAM & 0.29 & Trust & 0.21 \\
\hline 4 & Big Data & 18 & Adoption & 0.28 & Big Data & 0.21 \\
\hline 5 & $\begin{array}{l}\text { Business } \\
\text { Model }\end{array}$ & 17 & e-Government & 0.27 & e-Commerce & 0.22 \\
\hline 6 & Adoption & 15 & Trust & 0.25 & Adoption & 0.23 \\
\hline 7 & e-Business & 15 & Big Data & 0.22 & e-Government & 0.23 \\
\hline 8 & Trust & 13 & Facebook & 0.13 & Motivation & 0.24 \\
\hline 9 & Smart Cities & 12 & Open Data & 0.09 & Literature Review & 0.26 \\
\hline 10 & TAM & 12 & Twitter & 0.09 & $\begin{array}{l}\text { Technology } \\
\text { adoption }\end{array}$ & 0.26 \\
\hline
\end{tabular}

Finally, core-periphery analysis was performed to identify the core research topics, from a whole-network perspective, as individual keywords, regardless of the cluster they belong to. The analysis yielded 10 topics in each of the categories (Table 2):

- Popularity: how frequently a keyword is used;

- Coreness: how connected is a keyword with other topics; value range: [0-1];

- Constraint: how connected is a keyword with other otherwise distinct topics (i.e., if the topic creates a backbone of the field); constraint is measured on a [0-1] scale.

High core value indicates a topic that is well connected to other topics. Lower constraint suggests a keyword that brings together otherwise isolated topics ("bridges"). Burts constraint (i.e., Constraint) [3] is commonly used for this purpose (accurately speaking, the lack of it, because the larger the constraint, the less structural opportunities a node may have for bridging structural holes). Topics with high Popularity and Coreness and low Constraint can be considered as driving forces for advancements in the field: without these topics, the field of I3E would be completely fragmented.

\section{Discussion and Conclusions}

The I3E conference on e-Business, e-Services and e-Society has grown and evolved over the years. The mere fact that the conference has managed to keep and expand its active community of researchers for almost two decades, demonstrates that the field is of increased and growing relevance. The objective and contribution of this paper is twofold: (a) to have a methodological contribution in the field (i.e., the method presented in this paper has not been employed in this research domain before); and (b) to demonstrate and apply a quantified (objective) methodology that is beyond the qualitative (subjective) perspective of a systematic literature review, aiming to shed light to the maturation of the field throughout the past two decades and to map the research 
streams in the field, how they are connected, and detect those that would benefit for more focus or integration. The analysis conducted in this study highlighted the major themes that have dominated the researchers' interest, as well as those that are likely to be the core focus of future studies. The co-word analysis of the $\mathrm{I} 3 \mathrm{E}$ proceedings to date revealed some interesting findings regarding the progression of the field as a whole.

In coherence with the title of the conference, the three main (i.e., motor) themes have been on e-business (i.e., e-commerce), e-services (i.e., e-government) and e-society (i.e., social media) as is depicted in Fig. 5, and in line with previous systematic literature reviews $[2,26]$. The underlying topic that has linked these three focus areas has been adoption models, examining the factors concerning individuals, users and organizations intention to adopt such systems, also noted in previous studies $[8,1]$. This trend also indicates the maturity of these technologies, with substantial research still concerning why and how adoption of emerging digital technologies can be enhanced.

An intriguing finding is the emergence of big data as a linkage between the main three pillars (Figs. 4 and 5). Research themes that fall within the "Chaos/Unstructured" quadrant will attract attention in the years to come: as more and more people are moving from the adoption of digital technologies to the routinization in everyday life, so is the amount of generated data increasing. This has led to a surge of research on the potential of big data analytics for extracting actionable insight confirming previous findings [22].

As seen from the network map (Fig. 5), big data - in particular with relevance to egovernment and social media - is an area with much future interest, also highlighted in [13]. The use of user generated data, and the integration with social media platforms offers an interesting perspective into the generated insight, the services that can be built based on this insight, as well as the implications and ethical issues that accompany such ventures. Big data and business analytics ecosystems may pave the way towards digital transformation and sustainable societies [23]. Furthermore, the strong link between e-commerce and social media (Table 1), denotes the increased prevalence of social commerce efforts over the last years (e.g., [18]). We witness an increased number of social media platforms featuring commercial aspects, with some notable examples being Facebook and Instagram. As more vendors utilize these platforms, trust emerges as a core aspect [9], both regarding the sales side and the products featured on these, as well as on the data exchanged and utilized for marketing and sales promotions [8]. Recent events (e.g. the Cambridge Analytica scandal) surfaced issues about privacy violation and use of data by third-parties which have come to the attention of the general population. As social media and e-commerce vendors become increasingly more integrated, data sharing and ownership issues will be brought in the spotlight of research/practice.

While these are some of the major trends that can be detected throughout an analysis of the field covered by the I3E conference, there are many more sub-fields that are likely to be fruitful areas for future research. Our ambition was to illustrate some of these as well as to identify the core areas that have received the center light of attention by researchers over the past two decades. Our analysis indicates that the three pillars of the $\mathrm{I} 3 \mathrm{E}$ conference are becoming increasingly more fussed, and that future research is likely to bridge all three domains to derive important research and practical knowledge. 


\section{References}

1. Al-Sharhan, S.A., et al. (eds.): I3E 2018. LNCS, vol. 11195. Springer, Cham (2018). https:// doi.org/10.1007/978-3-030-02131-3

2. Alalwan, A.A., Rana, N.P., Algharabat, R., Tarhini, A.: A systematic review of extant literature in social media in the marketing perspective. In: Dwivedi, Y.K., et al. (eds.) I3E 2016. LNCS, vol. 9844, pp. 79-89. Springer, Cham (2016). https://doi.org/10.1007/978-3319-45234-0_8

3. Burt, R.S.: Structural holes and good ideas. Am. J. Sociol. 110(2), 349-399 (2004)

4. Callon, M., Courtial, J.P., Laville, F.: Co-word analysis as a tool for describing the network of interactions between basic and technological research: the case of polymer chemsitry. Scientometrics 22(1), 155-205 (1991)

5. Callon, M., Courtial, J.P., Turner, W.A., Bauin, S.: From translations to problematic networks: an introduction to co-word analysis. Inf. (Int. Social Science Council) 22(2), 191235 (1983)

6. Cambrosio, A., Limoges, C., Courtial, J., Laville, F.: Historical scientometrics? Mapping over 70 years of biological safety research with coword analysis. Scientometrics 27(2), 119143 (1993)

7. Cobo, M.J., López-Herrera, A.G., Herrera-Viedma, E., Herrera, F.: Science mapping software tools: review, analysis, and cooperative study among tools. J. Am. Soc. Inf. Sci. Technol. 62(7), 1382-1402 (2011)

8. Douligeris, C., Polemi, N., Karantjias, A., Lamersdorf, W. (eds.): I3E 2013. IAICT, vol. 399. Springer, Heidelberg (2013). https://doi.org/10.1007/978-3-642-37437-1

9. Grover, P., Kar, A.K., Vigneswara Ilavarasan, P.: Blockchain for businesses: a systematic literature review. In: Al-Sharhan, S.A., et al. (eds.) I3E 2018. LNCS, vol. 11195, pp. 325336. Springer, Cham (2018). https://doi.org/10.1007/978-3-030-02131-3_29

10. He, Q.: Knowledge discovery through co-word analysis. Libr. Trends 48, 133-159 (1999)

11. Hu, C.P., Hu, J.M., Deng, S.L., Liu, Y.: A co-word analysis of library and information science in china. Scientometrics 97(2), 369-382 (2013)

12. Ismagilova, E., Slade, E., Williams, M.: Persuasiveness of ewom communications: literature review and suggestions for future research. In: Dwivedi, Y.K., et al. (eds.) Social Media: The Good, the Bad, and the Ugly, pp. 354-359. Springer, Cham (2016). https://doi.org/10.1007/ 978-3-319-45234-0_32

13. Janssen, M., et al. (eds.): I3E 2015. LNCS, vol. 9373. Springer, Cham (2015). https://doi. org/10.1007/978-3-319-25013-7

14. de Laat, M., Lally, V., Lipponen, L., Simons, R.J.: Investigating patterns of interaction in networked learning and computer-supported collaborative learning: arole for social network analysis. Int. J. Comput.-Support. Collab. Learn. 2(1), 87-103 (2007)

15. Leydesdorff, L., Vaughan, L.: Co-occurrence matrices and their applications in information science: extending ACA to the web environment. J. Am. Soc. Inf. Sci. Technol. 57(12), 1616-1628 (2006)

16. Li, H., Mäntymäki, M., Zhang, X. (eds.): I3E 2014. IAICT, vol. 445. Springer, Heidelberg (2014). https://doi.org/10.1007/978-3-662-45526-5

17. Liu, Y., Goncalves, J., Ferreira, D., Xiao, B., Hosio, S., Kostakos, V.: CHI 1994-2013: mapping two decades of intellectual progress through co-word analysis. In: Proceedings of the 32rd Annual ACM Conference on Human Factors in Computing Systems, pp. 3553 3562. ACM, New York (2014) 
18. Mikalef, P., Pappas, I.O., Giannakos, M.N., Sharma, K.: Determining consumer engagement in word-of-mouth: trust and network ties in a social commerce setting. In: Kar, A.K., et al. (eds.) I3E 2017. LNCS, vol. 10595, pp. 351-362. Springer, Cham (2017). https://doi.org/10. 1007/978-3-319-68557-1_31

19. Murtagh, F., Legendre, P.: Ward's hierarchical agglomerative clustering method: which algorithms implement ward's criterion? J. Classif. 31(3), 274-295 (2014)

20. Newman, M.E.: A measure of betweenness centrality based on random walks. Soc. Netw. 27(1), 39-54 (2005)

21. Nielsen, A.E., Thomsen, C.: Sustainable development: the role of network communication. Corp. Soc. Responsib. Environ. Manag. 18(1), 1-10 (2011)

22. Pappas, I.O., Mikalef, P., Dwivedi, Y.K., Jaccheri, L., Krogstie, J., Mäntymäki, M. (eds.): I3E 2019. LNCS, vol. 11701. Springer, Cham (2019). https://doi.org/10.1007/978-3-03029374-1

23. Pappas, I.O., Mikalef, P., Giannakos, M.N., Krogstie, J., Lekakos, G.: Big data and business analytics ecosystems: paving the way towards digital transformation and sustainable societies. IseB 16(3), 479-491 (2018). https://doi.org/10.1007/s10257-018-0377-z

24. Rombach, P., Porter, M.A., Fowler, J.H., Mucha, P.J.: Core-periphery structure in networks (revisited). SIAM Rev. 59(3), 619-646 (2017)

25. Schank, T., Wagner, D.: Approximating clustering-coefficient and transitivity. Universität Karlsruhe, Fakultät für Informatik (2004)

26. Sorrentino, M., Niehaves, B.: Intermediaries in e-inclusion: a literature review. In: 2010 43rd Hawaii International Conference on System Sciences, pp. 1-10 (2010)

27. Tamilmani, K., Rana, N.P., Prakasam, N., Dwivedi, Y.K.: The battle of brain vs. heart: a literature review and meta-analysis of hedonic motivation use in UTAUT2. Int. J. Inf. Manag. 46, 222-235 (2019) 